\title{
UN CONCEPTO ESTRUCTURAL DE APROXIMACIÓN EMPÍRICA
}

\author{
C. ULISES MOULINES \\ Universidad Nacional \\ Autónoma de México
}

\section{Introducción}

El objetivo de este artículo es precisar formalmente un concepto de aproximación que sea relevante para la filosofía de ciencia. Quizás se piense que ésta es una tarea ya resuelta hace tiempo. En efecto, en la topología moderna podemos hallar una definición matemática exacta del concepto de aproximación. Sin embargo, esto no respondería al problema que vamos a plantear aquí. Es indudablemente cierto que la teoría de los espacios topológicos permite una caracterización exacta de la aproximación en matemáticas y también considero cierto que cualquier ulterior investigación metodológica debe basarse en las definiciones matemáticas. Pero esas definiciones de la topología por sí mismas no nos proporcionan indicación alguna acerca de la forma lógica y la función que tienen las relaciones aproximativas dentro de las ciencias empíricas. Y esta última cuestión es un problema específico de la filosofía de la ciencia (aunque para su resolución hagamos uso de instrumentos topológicos).

Casi nadie dudará hoy día que las ciencias empíricas, incluidas las llamadas ciencias "exactas" como la física, no son precisamente exactas, sino que sólo concuerdan con la experiencia de manera aproximada.' Pero aunque nadie

1 No voy a plantearme aquí la pregunta de moda sobre "si puede existir una experiencia sin teoria" para darle la respuesta de moda: "no; todo es teoría". A mi entender, esa pregunta y su respuesta usual son sumamente ambiguas y confundentes. Sólo quiero señalar que presupongo que las teorías científicas no son mónadas leibnizianas ocupadas sólo en la contemplación narcisista de sí mismas, sino que suelen chocar con algo externo a ellas a lo que podemos 
dude de este hecho, en cambio suele olvidarse que el carácter aproximativo es un rasgo esencial de la estructura de las ciencias empíricas que debería, por tanto, tenerse muy en cuenta en el análisis lógico de la ciencia.

La filosofía de la ciencia ha descuidado ese aspecto hasta hace poco. La negligencia ha sido debida quizás a que se suponia que aquí no radica ningún problema filosóficamente relevante, o bien a que se consideraba la problemática como técnicamente demasiado complicada para darle un tratamiento fructífero.

La situación ha cambiado en años recientes. Diversos autores, desde diversos puntos de partida, han hecho interesantes esfuerzos por clarificar la problemática de la aproximación empírica. Los trabajos más sistemáticos se han hecho en Alemania y Polonia, principalmente.

Aquí sólo voy a referirme a dos intentos que me parecen especialmente notables. En primer lugar, tenemos la propuesta de E. Scheibe de introducir un concepto de explicación aproximativa de leyes o teorías como modificación más realista de la elucidación anterior del concepto de explicación propuesta por Hempel (cf. [8]). Scheibe aplicó su concepto al caso concreto de la relación entre las leyes de Kepler y la teoría de la gravitación de Newton: según Scheibe, la segunda explica aproximativamente las primeras (cf. [7]). Recientemente, M. Käsbauer ha mostrado en un análisis formal del concepto general de Scheibe (cf. [2]), que éste no puede considerarse propiamente como una explicación, dado que no cumple una condición esencial para cualquier relación lógica entre enunciados que pretenda ser una explicación: la asimetría deductiva entre explanans y explanandum. A pesar de esta grave dificultad, el análisis que proporciona Scheibe de la relación entre la ley de Newton y las de Kepler sigue siendo interesante como elucidación de

dar el nombre genérico de "experiencia". Este choque puede ser lo bastante fuerte como para convertirse en catástrofe. Y uno de los "trucos" que los científicos emplean para evitar esas catástrofes es justamente el concepto de aprorimación. 
un caso de relación interteórica aproximativa, aunque esta relación no pueda considerarse como explicación en sentido estricto.

Una propuesta más general que la de Scheibe para precisar el concepto de aproximación es la de G. Ludwig. A pesar de ser una de las propuestas más interesantes existentes hasta la fecha ha permanecido prácticamente ignorada por los medios filosóficos. (Esto acaso se deba a dos razones: primera, a que la propuesta de Ludwig aparece dentro de un libro aparentemente especializado de física; segunda, y más importante, a que el libro está escrito en alemán. . . dos graves obstáculos para muchos filósofos actuales.)

En el segundo capítulo de su libro [3], Ludwig desarrolla un marco general y sumamente abstracto para la reconstrucción de las teorías físicas. Ese marco es interesante por sí mismo como metateoría formal de las teorías físicas; pero es particularmente interesante en el presente contexto pues en él tiene cabida una noción elaborada y muy general de aproximación: se trata de la proporcionada por el método de "emborronar mediante principios de representación imprecisos" ("durch unscharfe Abbildungsprinzipien verschmieren") las relaciones lógico-matemáticas que operan dentro de una teoría o entre teorías distintas. Aquí no vamos a exponer con detalle la metateoría general de Ludwig; esperamos poder hacerlo en un trabajo posterior sobre relaciones interteóricas aproximativas, un tema fundamental en la obra de Ludwig. Baste notar aquí que las ideas básicas de Ludwig sobre el funcionamiento de la aproximación han ejercido una influencia considerable en el presente trabajo, aunque los aparatos conceptuales utilizados en uno y otro caso difieran en gran medida, así como los detalles de interpretación.

El postulado metodológico de partida que admitimos aquí es la necesidad de incluir una o más nociones de aproximación en cualquier programa de reconstrucción lógica de teorías empíricas que pretenda ser completo. En un programa tal se trata de dar cuenta de las relaciones entre una teoría 
y su campo de experiencia (o campo de aplicaciones), y de las relaciones entre una teoría y otra. $Y$ en ambos tipos de relaciones intervienen, sin duda, componentes aproximativos.

En los últimos años ha cuajado un programa de reconstrucción lógica (o "metateoría", si se quiere) de teorías empíricas, al que Y. Bar-Hillel bautizó "concepción estructural de las teorías". 2 Este programa fue iniciado por J. D. Sneed en [9] y ha sido ulteriormente reformulado, desarrollado y aplicado por W. Stegmüller (cf. [11], [12]), por el propio Sneed (cf. [10]) y por otros, entre ellos el presente autor (cf. [5]).

Hasta ahora, la concepción estructural no había tomado en consideración los aspectos aproximativos de las teorías empíricas. En la medida en que dicha concepción aspire a procurar una imagen integral de la estructura y funcionamiento de las teorías empíricas debe incluir un análisis y reconstrucción de aquellos aspectos. El presente artículo contiene un primer paso en esa dirección.

\section{Brevísimo esbozo de la concepción estructural}

Para discutir de qué modo ha de incluirse un componente aproximativo en el programa sneediano de reconstrucción de teorías, debe estar previamente clara la forma lógica que según esa concepción tienen una teoría y su contenido empírico. Se presupone que el lector posee un conocimiento mínimo de los conceptos básicos del programa. Aquí no podemos entrar en detalles. ${ }^{3}$ Sólo vamos a mencionar los aspectos más relevantes para nuestro propósito.

2 El calificativo "estructural" no indica ninguna conexión íntima con las corrientes llamadas "estructuralistas" en lingüistica, etnología y en la culturilla difusa de los países latinos, salvo quizás el rasgo trivial de dar importancia a la consideración de estructuras globales en el análisis del conocimiento.

3 Estos conocimientos puede adquirirlos ya sea en las obras originales [9], [11], ya sea en comentarios más recientes. En castellano pueden leerse los artículos [4], [13] y [14]. 
Según la metateoría estructural, no es conveniente concebir una teoría empírica meramente como un conjunto de enunciados, siguiendo las concepciones más corrientes (statement view); una teoría posee una estructura más compleja. En esencia una teoría $T$ consiste en un par ordenado de entidades determinadas modeloteóricamente: un núcleo matemático $N$ y un dominio de aplicaciones empíricas propuestas $I$, que incluye los "pedazos de realidad" que efectivamente intentamos explicar mediante la teoría. La razón por la que se incluye el campo de aplicaciones $I$ en el concepto mismo de teoría y no se le considera como algo externo a ella es que se toman en serio las vagas insinuaciones de Kuhn y otros, según las cuales no se ha identificado una teoría empírica si, además de su estructura conceptual (matemática), no se conocen también (unos cuantos o todos los) casos a los que se intenta aplicar esa estructura conceptual.

La distinción entre $N$ e $I$ está indirectamente relacionada (aunque no por una relación de necesidad lógica) con otra dicotomía importante dentro de la concepción estructural: la distinción entre conceptos teóricos y conceptos no-teóricos relativamente a una teoría $T$. Esta distinción no tiene (casi) nada que ver con la dicotomía tradicional entre lenguaje observacional y lenguaje teórico. La distinción entre conceptos teóricos y no-teóricos es relativa a cada teoría. Además, no es una distinción semántica ni ontológica, sino puramente funcional: atiende a como "funcionan", a como "se manejan" los conceptos dentro de una teoría dada. En una caracterización más general que la original de Sneed, podemos decir que los conceptos $T$-no-teóricos son aquellos que se emplean o "manipulan" en teorías previas a e independientes de $T$. El uso de los conceptos $T$-teóricos, en cambio, presupone la aceptación de $T$. Los conceptos $T$-no-teóricos, al no presuponer $T$, constituyen, por asi decir, su base confirmatoria o "empírica" (en sentido relativo). Por eso, el campo de aplicaciones $I$ viene determinado exclusivamente por conceptos no-teóricos. 
El núcleo $N$ de $T$ está formado, a su vez, por varias entidades modeloteóricas:

$$
N=\left\langle M_{\mathrm{p}}, M_{\mathrm{pp}}, r, M, C>,\right.
$$

donde $M_{\mathrm{p}}$ es el conjunto de modelos posibles de la teoría (estructuras que contienen todos los conceptos de la teoría, pero no especifican las leyes que los relacionan entre sí); $M_{\mathrm{pp}}$ es el conjunto de modelos posibles parciales de la teoría (estructuras que sólo contienen los conceptos $T$-no-teóricos); $r$ es una función que a cada modelo de $M_{\mathrm{p}}$ le asigna uno en $M_{\mathrm{pp}}$ (el que le corresponde al primero al quitarle, por así decir, los conceptos $T$-teóricos); $M$ es el conjunto de modelos efectivos de la teoría (modelos posibles que además satisfacen realmente las leyes fundamentales de la teoría); y $C$ es un conjunto de condiciones llamadas de "ligadura" (en inglés: constraints; en alemán: Nebenbedingungen), representadas también modeloteóricamente, y que conectan entre sí los modelos mediante restricciones hechas a los conceptos que se corresponden en modelos distintos.

El campo de aplicaciones $I$ es un subconjunto propio del conjunto de los modelos posibles parciales: $I \subset M_{\mathrm{pp}}$.

El núcleo de una teoría avanzada generalmente posee "ramificaciones" o "prolongaciones" más especiales y más sujetas a revisión: se trata de las leyes especiales y las condiciones especiales de ligadura que se van añadiendo o quitando al núcleo, según las necesidades empíricas; este proceso constituye la "evolución normal" de la teoría en el sentido de Kuhn. El núcleo más las leyes y ligaduras especiales añadidas en un estadio determinado de la evolución de la teoría constituyen un "núcleo expandido" o "expansión" E:

$$
E=<M_{\mathrm{p}}, M_{\mathrm{pp}}, r, M, C, L, C_{\mathrm{L}} ; \alpha>,
$$

donde $L$ representa el conjunto de leyes especiales admitidas en ese estadio $(L \subset M) ; C_{\mathrm{L}}$ el conjunto de ligaduras especiales y $\alpha$ es una relación que especifica para cada apli- 
cación propuesta las leyes especiales que le corresponden y que se intenta que la expliquen. ${ }^{4}$

La evolución normal de la teoría consiste en una sucesión de $E$ s que en el caso ideal contendrán cada vez más leyes especiales con cada vez más aplicaciones conocidas. Y el contenido empírico de la teoría correspondiente a un núcleo expandido $E$ se expresa como un enunciado global (el enunciado empirico central de la teoria) que asevera la aplicabilidad de $E$ como estructura global al campo de aplicaciones $I$ en su totalidad. Simbólicamente:

$$
I \in A(E),
$$

donde $A$ es una operación de "aplicación global" definida a partir de las relaciones $\alpha$ y $r$.

Bien, nuestro problema es: ¿Cómo se inserta coherentemente una estructura aproximativa en ese cuadro general?

\section{Casuística de la aproximación empírica}

No hay razón para suponer que exista una respuesta unívoca a la anterior pregunta. Sería un prejuicio creer de buenas a primeras que todos los aspectos aproximativos entran bajo la misma forma lógica en la reconstrucción de las teorías empíricas. Más bien hemos de suponer que el uso aproximativo de las teorías ofrece aspectos lógicamente bien diferenciados. Por mi parte, veo por lo menos dos niveles netamente distintos.

En primer lugar hemos de considerar un nivel que podríamos llamar "puramente empírico" o "preteórico" (relativamente, claro, a una teoría dada $T$ ). Se trata de la sistematización matemático-conceptual de los datos "obser-

4 Recientemente, Sneed y W. Balzer han creado una terminología y un formalismo más elegantes, y quizás también potentes, para representar formalmente las relaciones entre $N$ y los diversos $E$ s sucesivos. El nuevo concepto central es el de red de (mini)teorías (cf. [10], por ejemplo). Pero dado que este nuevo formalismo no añade nada radicalmente nuevo al anterior y aún no ha sido publicado, me atengo al formalismo original de Stegmüller. 
vacionales" que se han obtenido sobre un determinado dominio empírico al que se intenta aplicar la teoría; en la terminología estructural, se trata de la construcción de los modelos posibles parciales de $T$.

Está claro que este primer paso, previo a la aplicación misma de la teoría, supone el uso de métodos de aproximación. Se trata, por ejemplo, de los métodos de la física experimental que pérmiten construir "modelos" cuantitativos simplificados de los datos obtenidos; pongamos por caso la determinación matemática de la órbita de un planeta partiendo de un número finito de observaciones astronómicas.

Este primer nivel de aproximación no es el que vamos a tratar aquí. La razón es que sobre él no tengo nada que decir que sea formalmente preciso o intuitivamente interesante. Aquí vamos a tratar un segundo nivel de aproximación que es, por así decir, de carácter más teórico. Es el que consiste en la transición de los modelos parciales (o "empíricos") a los modelos efectivos de la teoría. Este es el paso que permite establecer el enunciado empírico central de la teoría, es decir, el enunciado que asevera que los modelos parciales construidos en el estadio preteórico pueden ser subsumidos efectivamente bajo la teoría; dicho de otro modo, el enunciado que asevera que la teoría puede aplicarse efectivamente a los modelos parciales dados. Ahora bien, esa aplicación casi nunca se logrará de manera exacta. Claro que un modelo parcial dado puede ser convertido trivialmente en un modelo posible, simplemente añadiéndole los conceptos teóricos requeridos; pero ese modelo posible casi nunca será exactamente el modelo efectivo que deseábamos y que había de cumplir determinadas leyes de la teoría.

Supongamos, por ejemplo, que después del primer proceso de aproximación antes reseñado concebimos el sistema físico real constituido por la Tierra, la Luna y el Sol como un sistema idealizado constituido por tres partículas que se mueven sobre curvas continuas. Este primer paso aproximativo todavía no nos garantiza que ese sistema idealizado sea 
subsumible bajo la teoría de la gravitación de Newton, o sea, no nos garantiza que la teoría de la gravitación sea efectivamente aplicable a dicho sistema. Históricamente, este fue uno de los problemas centrales de la mecánica celeste durante el siglo xviI, y, hasta Laplace, el grado de aproximación con que se aplicaba la teoría de la gravitación al sistema Tierra-Luna-Sol (idealizado) fue bastante grosero. Este es sólo un ejemplo entre miles.

En el segundo capítulo de su libro, Sneed discute brevemente el problema de la validez aproximada de las teorías físicas; su opinión es que puede hacerse abstracción de ese problema si ya se toman los modelos parciales como "idealizaciones" (aproximaciones) de los datos empíricos. Pero esto no liquida el problema de las relaciones aproximativas. Pues acabamos de ver con el ejemplo anterior que ni siquiera fuertes idealizaciones de los datos empíricos tienen por qué concordar exactamente con las leyes de la teoría; y no por eso vamos a dejar de considerar que la teoría es aplicable - del mismo modo que los astrónomos del xviII no dejaron de considerar que la teoría de la gravitación era aplicable al sistema Tierra-Luna-Sol, a pesar de que sólo conseguían aproximaciones groseras. A la pregunta de si una teoría puede aplicarse a un dominio propuesto generalmente no puede responderse con un "sí" o un "no" categóricos. Hay que responder "más o menos" - y a lo sumo puede indicarse el grado de aplicabilidad.

Otro aspecto de la problemática de la aproximación que Sneed descuida es el de la aproximación como relación interteórica. Si admitimos que dentro de una determinada disciplina científica (por ejemplo, la mecánica o la genética) pueden identificarse diversas teorías en el sentido sneediano (por ejemplo, la mecánica clásica de partículas o la mecánica del sólido rígido; la genética mendeliana o la genética de poblaciones) entonces está claro que deben existir ciertas relaciones entre esas teorías. Sneed y Stegmüller han reconstruido dos tipos generales de relaciones interteóricas; a 
saber, equivalencia entre teorías y reducción de una teoría a otra. Las definiciones propuestas de equivalencia y reducción presuponen que se trata de relaciones exactas entre las teorías. Ahora bien, aunque en la actualidad disponemos todavía de pocos ejemplos concretos de relaciones interteóricas precisadas formalmente, no obstante, es de suponer que muchas de las supuestas reducciones sólo pueden representarse formalmente como relaciones aproximativas. Uno de estos casos parece ser el de la relación entre las leyes de Kepler y la teoría de la gravitación, tal como ha sido reconstruida por Scheibe en [7].

Un tratamiento sistemático tanto de las relaciones interteóricas aproximativas como de la aplicación aproximativa de teorias individuales requiere un aparato formal que precise la noción de aproximación en general y permita reconstruir los enunciados aproximativos dentro de una teoría o las relaciones aproximativas entre las teorias. En lo que sigue se propone un aparato formal semejante.

\section{El programa}

De lo que se trata es de precisar la noción de imprecisión, es decir, de introducir un concepto formal que exprese de manera precisa la imprecisión (aproximación) con que se manejan las teorías empíricas. Esta noción debe formar parte del concepto mismo de teoría puesto que, como hemos argüido, la imprecisión es inherente y esencial a las teorías empíricas.

Hemos visto que, según la concepción estructural en su versión originaria, una teoría es una estructura que consta de dos entidades:

$$
T=\langle N, I>
$$

Aquí proponemos añadir al concepto de teoría un tercer elemento, que represente la relación aproximativa que existe 
ineludiblemente entre $N$ e $I$ y que se emplea constantemente al trabajar con $T$ :

$$
T=\langle N, ?, I>\text {. }
$$

Hemos de buscar pues una entidad que responda a este interrogante.

También hemos dicho que el contenido empírico de $T$ se expresa en un enunciado "holista" de la forma

$$
I \in A(E),
$$

donde $E$ es una determinada expansión de $N$. Pero asimismo hemos dicho que una teoría (no-trivial) nunca, o casi nunca, se aplica exactamente al dominio que se intenta explicar. Esto significa que el enunciado [III] nunca, o casi nunca, será válido. Podría decirse, siguiendo un uso corriente en los manuales científicos, que [III] es aproximadamente válido. Pero esto es, en el mejor de los casos, una metáfora de sentido oscuro, y, en el peor, una barbaridad semántica. Si no queremos meternos a construir extrañas semánticas con extraños valores veritativos, debemos admitir que los enunciados descriptivos de cualquier lenguaje razonable son o verdaderos o falsos, pero no "más o menos verdaderos" ni "más o menos falsos".

No es la verdad o falsedad de [III] lo que es aproximado; en rigor, [III] será en la inmensa mayoría de los casos simplemente falso. Lo aproximado es la aplicabilidad de $E$ a $I$ o, dicho equivalentemente, la subsunción de $I$ bajo $E$. En general, $E$ no se aplicará exactamente a $I$; pero lo que podemos sostener (si nuestra teoría funciona bien) es que $E$ se aplica aproximadamente a $I$. Es decir, podemos aseverar el enunciado (exactamente) válido: "La aplicación de $E$ a $I$ es aproximada".

La forma lógica de este último enunciado no será la misma que la de [III]; pero ciertamente será análoga en algún sentido. Como abreviación formal escribiremos

$$
\text { I } \tilde{\epsilon} A(E) \text {, }
$$


paralelamente a como usualmente se escribe " $a \simeq b$ " para el análogo aproximativo de " $a=b$ ".

Es obvio que para elucidar la forma lógica de [IV] y dar un sentido preciso al signo " $\tilde{\epsilon}$ " deberemos echar mano de la estructura aproximativa que ha de reemplazar el interrogante en [II] (aunque podemos sospechar ya que no bastará con eso). Tenemos pues una doble tarea ante nosotros. Primero, hallar la estructura aproximativa que complete adecuadamente el concepto de teoría en el sentido de [II]. Luego, emplear esa estructura para reconstruir la forma lógica de [IV], es decir, del enunciado empírico central de la teoría. En el presente artículo vamos a concentrarnos en el primer punto. Sobre el segundo sólo se harán breves insinuaciones; un tratamiento más detallado y formal del segundo punto se hallará en [6].

\section{Cómo "emborronar" el concepto estructural de teoría}

La idea de incluir un componente aproximativo en el concepto mismo de teoría se retrotrae a Ludwig. Claro que Ludwig propone un concepto general de teoría que (por lo menos a primera vista) es bastante distinto del concepto sneediano. Pero esto no nos impide seguir las líneas básicas de su método."

El componente que vincula aproximativamente la estructura matemática de una teoría a sus aplicaciones es, según Ludwig, un "sistema de conjuntos imprecisores" ("System. von Unschärfemengen"). . Los conjuntos imprecisores que se

5 En realidad, a pesar de las diferencias superficiales, son asombrosas las similitudes "a nivel profundo" que pueden detectarse entre la concepción de Ludwig y la de Sneed, dos concepciones que surgieron simultánea e independientemente la una de la otra. La concepción de Ludwig también merece el calificativo de "estructural", aunque los detalles concretos aparezcan en un ropaje muy distinto y, justo es decirlo, menos transparente que en Sneed. Sería interesante un estudio comparativo de ambas concepciones. Pero ésta es otra historia.

B A falta de mejor término, propongo traducir el alemán "Unschärfemenge" y el inglés "fuzzy set" por el neologismo técnico "conjunto imprecisor" (es una entidad que hace las cosas imprecisas o vagas, que produce imprecisión o vaguedad). No tengo reparos en introducir neologismos cuando se trata de denotar conceptos nuevos. 
introducen en una teoría son los que permiten aplicar aproximativamente las leyes de la teoría a determinados dominios empíricos y los que le permiten a uno decir que "la teoría funciona más o menos bien". La propuesta de Ludwig es elucidar el concepto de conjunto imprecisor de la forma más general posible mediante la noción topológica de estructura uniforme. ${ }^{7}$

Nuestro punto de partida será esta idea básica de Ludwig, me refiero concretamente a la idea de incluir una estructura uniforme especial en el concepto mismo de teoría, con el fin de representar formalmente las relaciones aproximativas. Claro que habrá que modificar esencialmente el procedimiento de Ludwig ya sólo sea por el hecho de que se trata de complementar la metateoría de Sneed y no la del propio Ludwig.

Más adelante daremos nuestra definición formal de "conjunto imprecisor" como elemento de una estructura uniforme. Pero antes es importante obtener una intuición clara de la noción de conjunto imprecisor.

Si queremos hacer impreciso un punto señalado sobre el papel, lo emborronamos y lo convertimos en una mancha. Una mancha es un conjunto de puntos, y podemos decir que todos los puntos dentro de la mancha son semejantes o análogos entre sí, en el sentido de que forman parte de la mancha (tienen el mismo color, ubicación próxima entre sí, etc.). Por tanto, también podríamos concebir la mancha no como un simple conjunto de puntos sino como un conjunto de pares de puntos análogos; cada par de puntos de la mancha están entre sí en una relación de semejanza, dada por la mancha. En definitiva lo que hemos hecho al emborronar el punto originario ha sido convertirlo en un conjunto de pares de puntos. Si emborronamos mucho, la mancha contendrá muchos pares de puntos; si emborronamos poco, contendrá menos. Si todos los pares de puntos de una mancha están tam.

7 Un estudio detallado de las estructuras uniformes en general se hallará en el segundo capítulo de [1]. 
bién en otra mancha, es que la primera es menor o igual que la segunda.

Una mancha es una representación intuitiva de un conjunto imprecisor.

¿Cuál puede ser el sentido de emborronar un punto? Supóngase que el punto cumplía una función de localización; por ejemplo, estaba marcado sobre un mapa de la ciudad para señalar algún lugar de la misma. Si marcamos un punto para señalar el lugar $A$, queremos decir: el lugar $A$ se halla exactamente allí. Pero si ahora emborronamos el punto y lo convertimos en una mancha, entonces queremos decir: el lugar A se halla más o menos por ahi. Puede parecer que esto no ofrece ninguna ventaja sino sólo la desventaja de mayor imprecisión. Pero sí tiene una ventaja práctica importante: que no nos comprometemos tanto. En el primer caso, es muy probable que nuestra afirmación sea, en rigor, falsa; en el segundo caso, es menos probable que lo sea. Y a pesar de todo, esa afirmación "emborronada" sigue siendo útil (a menos que emborronemos demasiado).

Este proceso de "emborronamiento" se aplica innumerables veces en nuestra experiencia cotidiana, muchas veces de manera implícita, puesto que ya las expresiones lingüísticas que usamos son imprecisas. Ahora bien, dicho proceso no sólo ocurre en la experiencia cotidiana, sino también en las ciencias más maduras, a pesar de que en éstas los términos usados suelen ser precisos. Tampoco en ellas queremos comprometernos demasiado y emborronamos los "puntos" en "manchas" o conjuntos imprecisores para salvaguardar nuestras leyes y teorías al aplicarlas.

Los conjuntos imprecisores son conjuntos de pares de entidades, relaciones si se quiere. Si un par de entidades pertenece a un conjunto imprecisor, esto significa que ambas entidades son semejantes o cercanas entre sí en algún sentido; el grado mínimo de semejanza o de cercanía que poseen viene fijado precisamente por el conjunto imprecisor al que pertenecen. 
De esta noción general e intuitiva de conjunto imprecisor no se sigue todavía cuál ha de ser el tipo de entidades que lo constituyan —qué son los "puntos" cuyos pares constituyen la "mancha". La única exigencia obvia de buenas a primeras es que las entidades en cuestión han de ser homogéneas, es decir, han de pertenecer todas a la misma categoría lógica.

En el enfoque de Ludwig, las entidades que constituyen los conjuntos imprecisores tienen la forma lógica de $n$-tuplos de términos de una teoría. Esos términos pueden denotar individuos físicos (por ejemplo, partículas, lugares, instantes) o bien valores numéricos (por ejemplo, valores-masa expresados en gramos, distancias expresadas en metros, segundos). Por razones más bien técnicas que de principio, tal construcción de los conjuntos imprecisores mediante tuplos más o menos complicados hace que la manipulación formal de los conjuntos imprecisores sea pesada e inelegante, como puede comprobarse en el texto de Ludwig.

Por el contrario, las características formales del aparato sneediano posibilitan una construcción más simple y natural de los conjuntos imprecisores. En efecto, las unidades mínimas de análisis lógico de las teorías no son aquí términos, sino modelos posibles o parciales de las teorías, o sea, estructuras enteras. Las relaciones aproximativas deberían ser reconstruidas del mismo modo que el resto de las relaciones teóricas que analiza el programa estructural: como relaciones entre entidades modeloteóricas. Conviene entonces construir los conjuntos imprecisores como conjuntos de pares de modelos posibles (o, en su caso, parciales). ${ }^{8}$

Un modelo posible representa una descripción matemática posible de una situación empírica o, dicho más exactamente, una descripción matemática posible de las propiedades de un sistema real (físico, biológico, social). Desde un punto de vista puramente lógico, para cualquier sistema dado

${ }^{8}$ En la discusión subsiguiente veremos por qué no convendría tomar modelos efectivos de la teoría. 
preteóricamente existen innumerables descripciones matemáticas posibles. Las diversas descripciones matemáticas del sistema, es decir, sus distintos modelos posibles pueden divergir respecto de los valores numéricos que toman ciertas funciones; pero la divergencia no tiene por qué ser siempre de carácter numérico: las diferencias en las descripciones pueden tener un carácter no-cuantitativo (ejemplo: en un modelo posible de un sistema de partículas se considera que las partículas son físicamente indivisibles; en otro modelo posible del mismo sistema no se hace ese supuesto, y por lo demás las funciones métricas pueden tomar los mismos valores en ambos modelos).

Lo que importa tener en cuenta es, en cualquier caso, lo siguiente: frecuentemente quisiéramos expresar formalmente que dos descripciones matemáticas distintas del mismo sistema, aunque no-idénticas, son próximas entre sí, son casi iguales, se parecen, etc. En nuestra terminología modeloteórica significa esto: comparamos dos modelos posibles de la teoría y queremos decir que son semejantes en cierto grado. La semejanza o proximidad de los modelos la expresaremos por medio de conjuntos imprecisores definidos modeloteóricamente, diciendo que el par constituido por los dos modelos comparados pertenece a un determinado conjunto imprecisor.

En la reconstrucción de los enunciados empíricos de las teorías deben incluirse esos conjuntos imprecisores modeloteóricos que determinan su carácter aproximativo. $\mathrm{Al} \mathrm{em-}$ plear un cierto conjunto imprecisor $u$ en la aplicación de la teoría $T$ a un sistema físico $S$ estamos admitiendo que el modelo posible $a$ de $S$ construido a partir de los datos empíricos en el lenguaje de $T$, por un lado, y el modelo efectivo $b$ predicho por $T$ para $S$, por otro, no tienen por qué coincidir totalmente, ${ }^{9}$ pero sí tienen que formar un par que esté dentro de $u$ :

$$
<a, b>\epsilon u
$$

- Esa es la razón por la que no conviene limitar los conjuntos imprecisores a que sólo incluyan modelos efectivos de la teoría, como anunciamos en la 
de lo contrario pensamos que algo no funciona bien en esa aplicación especial de la teoría.

Sea, por ejemplo, el caso en que, a partir de ciertas observaciones astronómicas sobre los movimientos planetarios, se construye un modelo posible $a$ de la teoría de la gravitación (una descripción de los planetas en el lenguaje de la teoría de la gravitación). En general, este modelo no se adecuará exactamente a la ley de la gravitación. Pero podremos indicar otro modelo posible $b$ que sí sea modelo efectivo de la ley de la gravitación y que no diverja mucho de $a$. Para expresar esta situación escribiremos:

$$
a \in M_{\mathrm{p}}[G] \wedge b \in M[G] \wedge<a ; b>\epsilon u,
$$

donde $M_{\mathrm{p}}[G]$ es el conjunto de modelos posibles de la teoría de la gravitación, $M[G]$ el conjunto de sus modelos efectivos y $u$ es un determinado conjunto imprecisor admitido en la teoría de la gravitación, que fija el grado de aproximación con que se puede aplicar esa teoría al sistema planetario.

En muchos casos, los conjuntos imprecisores podrán ser determinados mediante ciertas condiciones expresables en el lenguaje de la teoría. Así, por ejemplo, en una teoría que utilizase la función $s$ de posición (de partículas en instantes) podría fijarse un conjunto imprecisor $u$ para esa función (por lo menos en ciertos dominios) mediante la siguiente condición:

Dados dos modelos (posibles o parciales) $x, x^{\prime}$ cualesquiera,

$$
\begin{aligned}
& <x, x^{\prime}>\in \text { u ssi } P_{\mathrm{x}}=P_{\mathrm{x}^{\prime}} \wedge T_{\mathrm{x}}=T_{\mathrm{x}^{\prime}} \wedge \\
& (p)(t)\left(/ s_{\mathrm{x}}(p, t)-s_{\mathrm{x}^{\prime}}(p, t) /<\delta\right),
\end{aligned}
$$

nota anterior. Los modelos efectivos de la teoría casi nunca se adecuarán exactamente a los datos empíricos; en general, a estos últimos sólo se adecuarán exactamente modelos posibles. 
donde $P_{\mathrm{i}}$ es un conjunto de partículas, $T_{\mathrm{i}}$ un intervalo temporal y $\delta$ un número real positivo fijo, que determina el grado de aproximación admitido en esa teoría, para la determinación de la posición.

En este caso podría "identificarse" el conjunto imprecisor $u$ simplemente con el número real $\delta$. Pero las cosas no siempre serán tan sencillas. En general, la condición que explícitamente determine el conjunto imprecisor de una teoría será una expresión larga y complicada compuesta de varios enunciados sobre diversas funciones y valores numéricos de aproximación. Ya un ejemplo tan sencillo como (V) muestra que el miembro derecho de la equivalencia tiene una estructura mucho más complicada que el miembro izquierdo. Es más: en muchos casos ni siquiera se considera que valga la pena esforzarse por hallar la expresión analítica explícita que fija el conjunto imprecisor; su determinación se abandona a las intuiciones preteóricas del científico.

Por ejemplo, en los libros de texto de física suele decirse que la expresión (la "ley") que fija el periodo de un péndulo simple,

$$
\mathrm{T}=2 \pi \sqrt{\frac{1}{\mathrm{~s}}}
$$

es sólo "aproximativamente válida", si el ángulo de oscilación es "suficientemente pequeño". Estas expresiones tan vagas significan que al aplicar (VI) a casos concretos, por ejemplo a un reloj de péndulo, toleraremos un cierto margen de divergencia entre el periodo predicho según (VI) (modelo efectivo) y el periodo observado o calculado por otros medios distintos de (VI) (modelo posible). Casi nadie especifica cuál es el margen (el "error" $\delta$ ) tolerable en distintas aplicaciones concretas; eso se deja a la venia del físico experimental.

Por otro lado, es obvio que el miembro derecho de (V), por ejemplo, contiene más información que el izquierdo so- 
bre el tipo de aproximación empleado; pero para investigaciones lógico-metodológicas de carácter general, en las que uno sólo se interesa por la forma lógica de los enunciados y relaciones aproximativas y no por su aplicación práctica, la expresión modeloteórica global de la izquierda de (V) será mucho más adecuada y manejable que una indicación concreta de funciones, de valores, de $\delta_{\mathbf{s}}$, etc.

Aparte de esa ventaja económico-metodológica en el uso de conjuntos imprecisores modeloteóricos en vez de sus correlatos analíticos, notemos otra ventaja de carácter distinto: la generalidad del concepto modeloteórico de conjunto imprecisor lo hace apto para aplicarlo al análisis de relaciones aproximativas en disciplinas en las que son esenciales conceptos no-cuantitativos, por ejemplo, en la biología y las ciencias sociales. ${ }^{10}$ También en estas disciplinas podrían expresarse con precisión, mediante la noción modeloteórica de conjunto imprecisor, las aplicaciones aproximativas no-cuantitativas de la teoría.

\section{Estructuras uniformes}

Para dar cuenta de la estructura aproximativa de las teorías usamos pues la noción intuitiva de conjunto imprecisor. Se trata ahora de darle a esa noción intuitiva una caracterización formal. Para ello emplearemos, como ya anunciamos, el concepto topológico general de estructura uniforme. ¿Por qué este concepto y no otro? La razón es la siguiente. El uso de los conjuntos imprecisores para dar cuenta de la aproximación empírica, tal como lo hemos discutido en $\S 4$, tiene los mismos rasgos formales que el uso de la aproximación en matemática pura. Estos rasgos formales han sido sistematizados y determinados en topología pura introduciendo

10 EI uso que se hace, por ejemplo, del concepto de genotipo, al determinar si dos organismos tienen o no el mismo genotipo, muestra que el enunciado "los organismos A y B tienen el mismo genotipo" es de carácter aproximativo no-cuantitativo. Lo mismo puede decirse de muchos conceptos psicológicos y sociológicos, que son burdamente aproximativos (temperamento, clase social, régimen político). 
el concepto de estructura uniforme. ${ }^{\text {II }}$ Entonces, podemos decir que aquí estamos haciendo topología aplicada, aplicada al estudio de las ciencias empíricas reconstruidas modeloteóricamente. Los "puntos" de la topología pura serán interpretados como modelos posibles, los pares de puntos "cercanos" serán pares de modelos posibles "semejantes" de una teoría empírica, y los entornos topológicos serán conjuntos imprecisores. Esta interpretación empírica de la topología pura es admisible si también admitimos que los conjuntos imprecisores deben considerarse como elementos de una estructura uniforme asociada a la teoría. Dado que hemos hallado conveniente introducir los conjuntos imprecisores como entidades modeloteóricas, también la estructura uniforme que los determina deberá estar definida modeloteóricamente.

Toda la serie de conjuntos imprecisores que se usan en aplicaciones concretas de la teoría y que han de permitir la construcción del enunciado central de la teoría en su forma aproximativa, (IV), deberán ser elementos escogidos de la estructura uniforme en cuestión. Es decir, para cada modelo parcial $i \in I$ que se proponga como aplicación de la teoría deberá haber un modelo posible $x_{\mathrm{i}}$ que sea extensión teórica de $i$ (es decir; tal que $r\left(x_{\mathrm{j}}\right)=i$ ), un modelo efectivo $x_{\mathrm{i}}^{\prime} \mathrm{de}$ la teoría, que satisfaga efectivamente la expansión $E, y$ ade$m a ́ s$ un conjunto imprecisor $u_{\mathrm{i}}$ perteneciente a la estructura uniforme asociada a la teoría, tal que cumpla:

$$
<x_{\mathrm{i}} ; x_{\mathrm{i}}^{\prime}>\epsilon u_{\mathrm{i}} \text {. }
$$

La conyunción coherente de todas estas aproximaciones en las aplicaciones particulares de la teoría es lo que constituye el enunciado aproximativo general

$$
I \tilde{\epsilon} A(E) \text {. }
$$

11 Recuérdese que el concepto de estructura uniforme se introduce en topología para caracterizar la noción intuitiva no-métrica de cercanía o aproximación entre "puntos" o elementos de un conjunto cualquiera. 
La estructura uniforme en sí misma, al igual que el núcleo $N$ y el campo de aplicaciones $I$, es un componente permanente e inmutable de la teoría. En cambio, los conjuntos imprecisores necesarios para la versión aproximativa del enunciado central (IV) variarán con el desarrollo de la teoría, pues el mismo enunciado (IV) es una entidad variable. Los cambios en la selección de los conjuntos imprecisores, lo mismo que los cambios en el contenido de (IV), vienen determinados por la introducción de nuevas leyes, por nuevas técnicas de cálculo o de observación, etc.

Vayamos, por fin, a la caracterización formal de una estructura uniforme modeloteórica. Para ello introducimos primero un poco de simbolismo.

Si $C$ es un conjunto, entonces $P o t(C)$ es el conjunto-potencia de $C$ y $\Delta(C)$ es la diagonal de $C$, es decir, el conjunto de todos los pares idénticos de elementos de $C$.

Si $R$ es una relación diádica, entonces

$R^{-1}=\{\langle x, y\rangle /\langle y, x\rangle \epsilon R\}$

$R^{0} R=R^{2}=\{\langle x, y>/ \exists z(\langle x, z\rangle \in R \wedge<z, y\rangle \epsilon R)\}$.

Sea $M_{\mathrm{p}}$ el conjunto de modelos posibles de una teoría y $M_{\mathrm{pp}}$ el conjunto de sus modelos parciales.

$D$ 1. $U$ es una estructura uniforme sobre $M_{\mathrm{p}}$ ssi:

(1) $\phi \neq U \subseteq \operatorname{Pot}\left(M_{p} \times M_{\mathrm{p}}\right)$

(2) $\left(u_{1}\right)\left(u_{2}\right)\left(u_{1} \in U \wedge u_{1} \subseteq u_{2} \supset u_{2} \in U\right)$

(3) $\left(u_{1}\right)\left(u_{2}\right)\left(u_{1} \in U \wedge u_{2} \in U \supset u_{1} \cap u_{2} \in U\right)$

(4) $(u)\left(u \in U \supset \Delta\left(M_{\mathrm{p}}\right) \subset u\right)$

(5) $(u)\left(u \in U \supset u^{-1} \in U\right)$

(6) (u) $\exists v\left(u \in U \supset v^{2} \subseteq u \wedge v \in U\right)$

$D$ 2: Una relación diádica $u$ sobre $M_{\mathrm{p}}$ es un conjunto imprecisor de $M_{\mathrm{p}}$ ssi existe una estructura uniforme $U$ sobre $M_{\mathrm{p}}$ tal que $u \in U$.

En vez de definir la estructura sobre $M_{\mathrm{p}}$, también podríamos haberlo hecho del mismo modo sobre $M_{\mathrm{pp}}$, y entonces 
tendríamos definidos conjuntos imprecisores de modelos parciales, en vez de conjuntos imprecisores de modelos posibles. Esta doble alternativa es relevante para cuestiones que se tra$\tan$ en [6].

La axiomatización del concepto de estructura uniforme presentada en $D I$ es esencialmente la misma (salvo ligeras modificaciones técnicas) que la propuesta por $\mathrm{N}$. Bourbaki en [1]. Sólo que aquí se ha interpretado la axiomatización abstracta de Bourbaki en el aparato modeloteórico de Sneed. Las tres primeras condiciones de definición de $D 1$ determinan el concepto de filtro; las tres últimas son específicas de una uniformidad.

Antes de concluir, conviene aclarar cuál es el sentido intuitivo de cada una de las seis condiciones de $D 1$ para la interpretación presente, es decir, para la aproximación en teorías empíricas. Con ello resultarán plausibles esas condiciones no sólo para el caso de la aproximación en matemática pura, sino también para el caso que nos ocupa.

En primer lugar, recuérdese que, como habíamos dicho al principio de este artículo, el uso de la aproximación en las ciencias empíricas puede entenderse como una "estrategia de inmunización" de la teoría frente a la experiencia. Para darle cierto grado de inmunidad a la teoría se introducen los conjuntos imprecisores al aplicarla. Esa estrategia de inmunización es un procedimiento legítimo, o al menos legitimado por la práctica científica. Si exigiéramos absoluta exactitud en las aplicaciones de la teoría (formalmente: si exigiéramos el uso exclusivo de la diagonal $\left.\Delta\left(M_{\mathrm{p}}\right)\right)$, nos encontraríamos muy pronto con una falsación de la teoría; y esto es una situación desagradable que, digan lo que digan los popperianos ortodoxos, la mayoría de los científicos que no son masoquistas prefiere evitar. Por eso se inmuniza la teoría hasta cierto punto mediante la introducción de una estructura uniforme, cuyos diversos elementos, los conjuntos imprecisores, pueden usarse en distintas aplicaciones de la teoría para emborronarlas en mayor o menor grado, según convenga y sea admisible. 
Con esta interpretación general en mente, aclaremos cada uno de los axiomas de $D$ l. El primero postula simplemente que la estructura uniforme introducida no es vacía, que vamos a usar efectivamente algunos conjuntos imprecisores. La interpretación de $D$ 1-(2) en el lenguaje de la inmunización es la condición cuasi-trivial de que si el conjunto $u_{1}$ inmuniza la teoría, ésta también será inmunizada por un conjunto $u_{2}$ mayor que incluya el primero (cuanta más imprecisión, más inmunización). $D$ 1-(3) significa que si se puede inmunizar la teoría tomando el conjunto $u_{1}$ y asimismo tomando $u_{2}$, entonces también se la puede inmunizar tomando ambos conjuntos a la vez, es decir, tomando su intersección. (Nótese que la intersección de dos conjuntos imprecisores nunca puede ser vacía: por $D$ 1-(4), la intersección siempre incluye por lo menos la diagonal.) $D \quad 1-(4)$ exige que la diagonal, es decir, la exactitud absoluta, esté incluida en cualquier conjunto imprecisor. También esto es intuitivamente satisfactorio: sea cual sea el conjunto imprecisor que estemos usando para inmunizar la teoría hasta cierto punto, en cualquier caso no deberemos excluir la posibilidad dichosa y fortuita de que se dé una aplicación exacta. El axioma $D$ 1-(5) es obvio en la presente interpretación: el orden en que aparezcan los modelos de un par comparado en cualquier conjunto imprecisor no tiene relevancia alguna para el grado de inmunización de la teoría. El último axioma, expresa una condición crítica: si $u$ es un conjunto imprecisor que inmuniza la teoría, entonces siempre existe otro conjunto imprecisor $v$ que sigue inmunizando la teoría, a pesar de ser dos veces más estricto (o "más exacto") que $u$. Se trata de un axioma "optimista", que ciertamente representa una idealización de la práctica científica. En efecto, implica la posibilidad de aplicar la teoría de forma cada vez más exacta o menos burda. (Sin embargo, nótese que no implica que vayamos a alcanzar nunca la diagonal, es decir, la exactitud absoluta.)

Con las observaciones anteriores creo haber hecho plausible la idea de que la entidad aproximativa que nos faltaba 
para completar la noción general de tecría empírica y cuya función debería ser conectar aproximativamente el núcleo matemático de la teoría con su campo de aplicaciones ha de ser simplemente una estructura uniforme $U$, tal como ha sido definida en $D$ 1. Podemos ahora sustituir el interrogante de (II) en $\$ 3$ por $U$ :

$$
T=\langle N, U, I\rangle \text {. }
$$

Este es el concepto estructural modificado de teoría que se propone aquí.

Naturalmente, dada una teoría concreta, no estaremos justificados en asociarle cualquier estructura uniforme $U$ que satisfaga las condiciones de $D 1$. A $U$ le deberemos poner ciertas restricciones, según sea el caso. Algunas de estas restricciones serán de carácter general, según el tipo general de teoría de que se trate. Otras restricciones tendrán sentido exclusivamente para la teoría particular que se esté reconstruyendo.

Un tipo muy general de teorías empíricas, precisamente el tipo analizado en el programa sneediano, consiste en aquellas en las que tiene sentido la distinción entre conceptos teóricos y no-teóricos, es decir, aquellas en las que hay que distin. guir entre modelos posibles y modelos parciales. Hemos visto antes que se podría definir una estructura uniforme para los modelos parciales análogamente a como se ha hecho para los modelos posibles, simplemente cambiando $M_{p}$ por $M_{\mathrm{pp}}$ en $D I-(I)$. Podemos pues asociar a la teoría una estructura uniforme a nivel teórico y otra a nivel no-teórico. Está claro entonces que ambas deben ser compatibles. $\mathrm{Y}$ para ello hay que demostrar primero que pueden serlo; es decir, hay que mostrar primero que la exigencia de compatibilidad entre los dos niveles de aproximación es satisfacible. Una prueba formal de esto aparece en [6].

En este artículo se ha esbozado un programa para la reconstrucción lógica de la aproximación empírica y se ha introducido formalmente el concepto básico de estructura uni- 
forme para llevar a cabo dicho programa. Pero debe quedar claro que la tarea aún por realizar es ingente, tanto al nivel de la metateoría general, como al de sus aplicaciones a la reconstrucción de teorías científicas concretas.

\section{REFERENCIAS}

[1] Bourbaki, N. Topologie générale. París: Hermann \& Cie., 1951.

[2] Käsbauer, M. "Ein Erklärungsbegriff für axiomatisierte Wissenschaften". Ponencia del Coloquio Zur Struktur und Entwicklung naturwissenschaftlicher Theorien. Universidad de Munich, noviembre de 1975.

[3] Ludwig, G. Deutung des Begriffs 'physikalische Theorie' und Grundlegung der Hilbertraumstruktur der Quantenmechanik durch Hauptsätze des Messens. Berlín-Nueva York-Heidelberg: Springer-Verlag, 1970.

[4] Moulines, C. U. "Hacia un nuevo concepto de teoría empírica", en Con vivium, Año 1973/II-No. 39 (Barcelona).

[5] Moulines, C. U. "A Logical Reconstruction of Simple Equilibrium Thermodynamics", en Erkenntnis, Vol. 9, No. 1, 1975.

[6] Moulines, C. U. "Approximative Application of Empirical Theories: A General Explication", en Erkenntnis, Vol. 10, No. 2, 1976.

[7] Scheibe, E. "Die Erklärung der Keplerschen Gesetze durch Newtons Gravitationsgesetz", en Einheit und Vielheit. Festschrift für Carl Friedrich v. Weizsäcker (comp. por E. Scheibe y G. Süssman), Göttingen, 1973.

[8] Scheibe, E. "Vergleichbarkeit, Widerspruch und Erklärung", en Philo. sophie und Physik (comp. por R. Haller), Braunschweig, 1975.

[9] Sneed, J. D. The Logical Structure of Mathematical Physics. Dordrecht: Reidel, 1971.

[10] Sneed, J. D. "Philosophical Problems in the Empirical Science of Science: A Formal Approach", en Erkenntnis, en vías de publicación.

[11] Stegmüller, W. Theorie und Erfahrung. 2. Halbband (Theorienstrukturen und Theoriendynamik). Berlín-Nueva York-Heidelberg: Springer-Verlag, 1973.

[12] Stegmüller, W. "A Combined Approach to the Dynamics of Theories. How to Improve Historical Interpretations of Theory Change by Applying Set Theoretical Structures", en Erkenntnis, en vías de publicación.

[13] Stegmüller, W. "Dinámica de teorías y comprensión lógica" (trad. de D. Quesada), en Teorema (España: Universidad de Valencia), Vol. IV/4, 1974.

[14] Stegmüller, W. "Estructuras y dinámica de teorías. Algunas reflexiones sobre J. D. Sneed y T. S. Kuhn" (trad. de A. Morones), en Diánoia (Universidad Nacional Autónoma de México), No. 21, 1975. 
The purpose of this article is to give a first step towards a general account of the logical form of approximation in empirical science. To this purpose, some topological and model-theoretic notions are used. More concretely, the topological concept of a uniform structure (a special kind of a filter) is interpreted empirically and applied to a model-theoretic representation of approximation in empirical theories.

The philosophical tenet assumed is that any significant empirical theory has an irreducibly approximative character. The application of laws and theoretical structures to a given empirical domain as well as the relationship between different laws or theoretical structures is usually approximative. Perfectly exact science is science fiction. Philosophers of science have unduly neglected this essential aspect of empirical science.

Only in recent times, some efforts have been made in order to clear up empirical approximation formally. Two authors may be mentioned in this respect: the German philosophers of science E. Scheibe and G. Ludwig.

Ludwig's work on approximation is relevant to the present article. His basic idea consists of introducing a formal notion of approximation into his theory concept. That is, the formal concept of an empirical theory is considered as incomplete unless it contains an approximative component as a mediating link between the theoretical structure and its empirical domain. This approximative component is defined as a uniform structure.

This basic idea of Ludwig's has been adopted in the present article. But Ludwig's concrete procedure has to be modified and reinterpreted. The reasons for this are, first, that the elements of Ludwig's uniform structures are too amorphous and unmanageable, and, secondly, that we have decided to use not Ludwig's general apparatus for reconstructing empirical theories, but rather the modeltheoretic apparatus of the so-called structuralistic view, first propounded by J. D. Sneed. Our approximative component is a modeltheoretically defined uniform structure, and its elements are modeltheoretic fuzzy sets consisting of pairs of models which intuitively approximate each other by some degree.

The final purpose of this article is to show how this modeltheoretic uniform structure may be adequately interpreted as empirical approximation and how it coherently fits into the Sneedian 
framework, which has not included any approximative component to date. This is done in Sections 4 and 5. After an intuitive discussion in Section 4, the improvement of the structuralistic theory concept by means of a uniform structure representing approximation is systematically expounded and explained in Section 5. Sections 4 and 5 are the central parts of the article.

Before that, some introductory remarks are made. Section 1 contains a very rough and short resume of the structuralistic view. In Section 2, a synoptic view of different approximation cases is offered. A distinction is made between two main kinds of empirical approximation: the approximative relationship between a theoretical structure and its domain of application (intra-theoretical approximation), and the approximative relationship between two different theories (inter-theoretical approximation). In Section 3, the program is stated: to develop a general apparatus which allows for a formal account of the two kinds of approximation mentioned above. Systematic application of the general apparatus to this double task is left to future work. Only some hints at the first part of the task (intra-theoretical approximation) are included in Section 5.

(Summary by C. Ulises Moulines) 